\title{
Joint Effects of Synchronization Errors of OFDM Systems in Doubly-Selective Fading Channels
}

\author{
Wen-Long Chin ${ }^{1}$ and Sau-Gee Chen (EURASIP Member) ${ }^{2}$ \\ ${ }^{1}$ Department of Engineering Science, National Cheng Kung University, Tainan 701, Taiwan \\ ${ }^{2}$ Department of Electronics Engineering and Institute of Electronics, National Chiao Tung University, Hsinchu 30050, Taiwan
}

Correspondence should be addressed to Wen-Long Chin, johnsonchin@pchome.com.tw

Received 26 July 2008; Revised 8 November 2008; Accepted 3 December 2008

Recommended by George Tombras

The majority of existing analyses on synchronization errors consider only partial synchronization error factors. In contrast, this work simultaneously analyzes joint effects of major synchronization errors, including the symbol time offset (STO), carrier frequency offset (CFO), and sampling clock frequency offset (SCFO) of orthogonal frequency-division multiplexing (OFDM) systems in doubly-selective fading channels. Those errors are generally coexisting so that the combined error will seriously degrade the performance of an OFDM receiver by introducing intercarrier interference (ICI) and intersymbol interference (ISI). To assist the design of OFDM receivers, we formulate the theoretical signal-to-interference-and-noise ratio (SINR) due to the combined error effect. As such, by knowing the required SINR of a specific application, all combinations of allowable errors can be derived, and cost-effective algorithms can be easily characterized. By doing so, it is unnecessary to run the time-consuming Monte Carlo simulations, commonly adopted by many conventional designs of synchronization algorithms, in order to know those combined error effects.

Copyright (c) 2008 W.-L. Chin and S.-G. Chen. This is an open access article distributed under the Creative Commons Attribution License, which permits unrestricted use, distribution, and reproduction in any medium, provided the original work is properly cited.

\section{INTRODUCTION}

Orthogonal frequency-division multiplexing (OFDM) is a promising technology for broadband transmission due to its high spectrum efficiency, and its robustness to the effects of multipath fading channels and impulse noises. However, OFDM systems are sensitive to synchronization errors.

There are three major synchronization errors, including the symbol time offset (STO), carrier frequency offset (CFO), and sampling clock frequency offset (SCFO) in OFDM systems. When the symbol time (ST) is not located in the intersymbol interference (ISI) free region, ISI is introduced. The time-selective channel, CFO, and SCFO will introduce additional intercarrier interference (ICI).

The effects of the synchronization errors had been studied in literature, for example, in [1-15]. For conciseness, we only discuss some representative works here. In [1], the signal-to-interference-and-noise ratio (SINR) is analyzed considering the effect of the $\mathrm{CFO}$ in time-selective channels; the work in [2] analyzes the effect of the STO in frequencyselective channels; the work in [3] also analyzes the effect of the STO without considering ISI; the work in [4] analyzes the effect of the Doppler spread; the work in [5] analyzes the effect of the CFO and SCFO in time-selective channels; the work in [6] only analyzes the effect of the STO in doubly-selective channels; the works in [7-9] analyze the synchronization errors separately in frequency-selective channels; the works in [10-12] analyze the effect of the CFO in frequency-selective channels; and the works in [1315] consider the combined effects of the STO and CFO in frequency-selective channels.

Some works $[1-6,8-15]$ only consider partial synchronization errors; some works $[1-9,12,13,15]$ separately consider synchronization errors; and some works [2, 6-14] only consider frequency-selective channels. In addition, the works in $[6,8,10,11,13,14]$ consider the STO, while assuming that the STO is small; therefore, nonnegligible ISI was often neglected. In summary, the current analyses mostly do not consider joint effects of the combined synchronization errors due to nonideal synchronization process in the environments of mobility (causing time-selective channel effect) and nonline-of-sight (NLOS) (causing multipath channel 
TABLE 1: Comparison of synchronization errors analyses.

\begin{tabular}{lcccccc}
\hline Reference & Consider ISI & Consider STO & Consider CFO & Consider SCFO & Fast fading channel & Combined analysis \\
\hline$[1]$ & No & No & Yes & No & Yes & No \\
{$[2]$} & Yes & Yes & No & No & No & No \\
{$[3]$} & No & No & No & No & Yes & No \\
{$[4]$} & No & No & Yes & Yes & Yes & No \\
{$[5]$} & Yes & Yes & No & No & Yes & No \\
{$[6]$} & No & Yes & Yes & Yes & No & No \\
{$[7]$} & Yes & Yes & Yes & Yes & No & No \\
{$[8]$} & No & Yes & Yes & Yes & No & No \\
{$[9]$} & No & No & Yes & No & No & No \\
{$[10,11]$} & No & Yes & Yes & No & No & Yes \\
{$[12]$} & No & No & Yes & No & No & No \\
{$[13]$} & No & Yes & No & No & No & Yes \\
{$[14]$} & No & Yes & Yes & No & No & No \\
{$[15]$} & No & No & Yes & No & Yes & Yes \\
This work & Yes & Yes & Yes & Yes & & \\
\hline
\end{tabular}

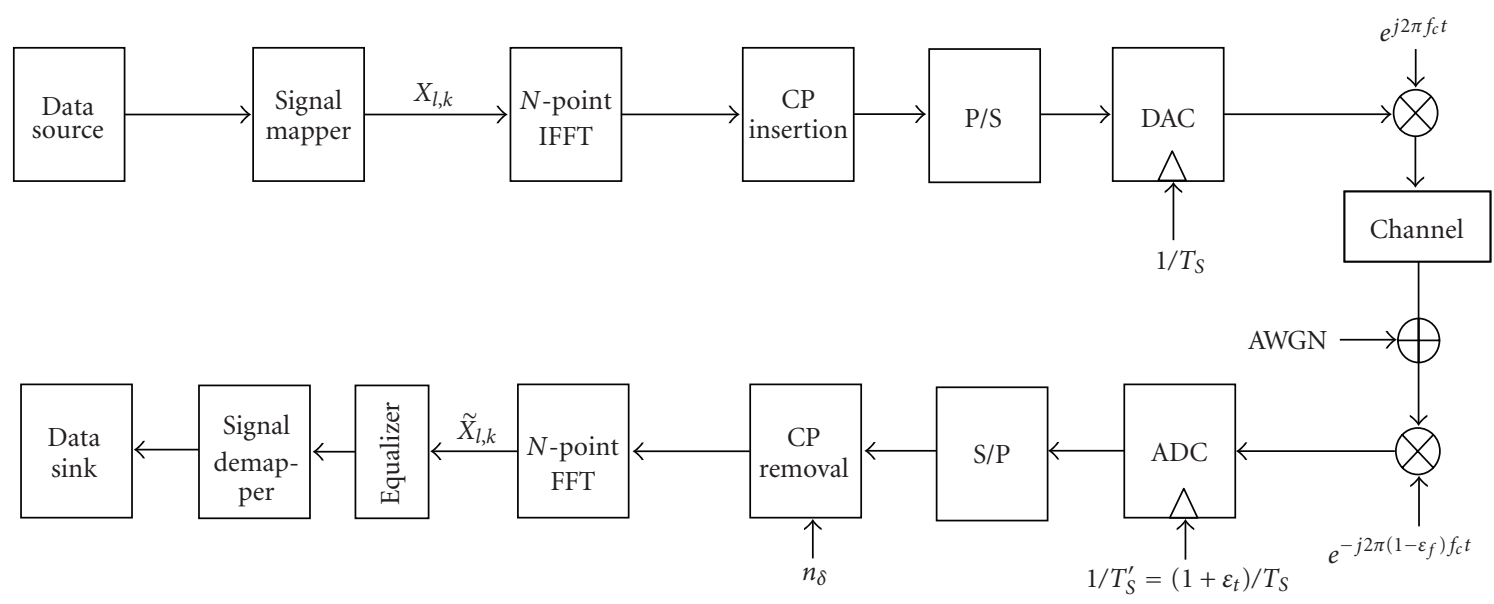

FIgURE 1: A simplified OFDM system model.

effect). For clarity, Table 1 summarizes the concerned errors and conditions, of some key representative works and the proposed work, on the synchronization error analyses.

The main contribution of this paper is that for better characterizations of synchronization errors under a practical communication environment, that is, in doubly-selective fading channels, we analyze joint effects of the mentioned three major synchronization errors, without the assumption of small STO. Another contribution is that compact forms can be derived from our work to gain further insights on the synchronization error effects. To this end, we first analyze the signal model of the combined synchronization errors in time-selective and frequency-selective fading channels by extending the works in [1-15]. Next, based on this model, the theoretical SINR is formulated. The derived SINR can be exploited to obtain all possible combinations of synchronization errors that meet the required SINR constraint, knowing that the allowable synchronization errors could help design suitable synchronization algorithms and shorten the design cycle. To gain further insights, some compact results are deduced from the derived SINR formulation. In addition, the works in $[1,2]$ are found to be special cases of this work; and our work is more accurate than that in [10].

The rest of this paper is organized as follows. The notations used in this work are summarized in the Notaions section at the end of the paper. The OFDM system model in doubly-selective fading channels is introduced in Section 2. The signal model with synchronization errors is analyzed, and its theoretical SINR is formulated in Section 3. Some compact results are given in Section 4. Numerical and simulation results are provided in Section 5. Finally, we conclude our work in Section 6.

\section{SYSTEM AND CHANNEL MODELS}

\subsection{System model}

In the following discussion, all the quantities indexed with $l$ belong to the $l$ th symbol. A simplified OFDM system 
model is shown in Figure 1. In this figure, $X_{l, k} / \tilde{X}_{l, k}$ is the transmitted/received frequency-domain data at the $k$ th subcarrier; $n_{\delta}$ is the STO; $1 / T_{S}$ is the transmitter's sampling frequency; $1 / T_{S}^{\prime}=\left(1+\varepsilon_{t}\right) / T_{S}$ is the receiver's sampling frequency, where $\varepsilon_{t}$ is the SCFO normalized by $1 / T_{S} ; \varepsilon_{f}$ is the CFO normalized by the subcarrier spacing; and $f_{c}$ is the carrier frequency. On the transmitter side, $N$ complex data symbols are modulated onto $N$ subcarriers by using the inverse fast Fourier transform (IFFT). The last $N_{G}$ IFFT output samples are copied to form the CP which is inserted at the beginning of each OFDM symbol. By inserting the CP, a guard interval is created so that ISI can be avoided and the orthogonality among subcarriers can be sustained. The receiver uses the fast Fourier transform (FFT) to demodulate received data.

\subsection{Channel model}

In this work, $h_{l}(n, \tau)$ denotes the $\tau$ th channel tap of the discrete time-selective CIR at time $n$ of the $l$ th symbol. Furthermore, the following two assumptions regarding the channels are made: (a) the channels are wide-sense stationary and uncorrelated scattering (WSSUS), and (b) the Doppler spectrum follows Jakes' model [16]. Based on these assumptions, the cross-correlation of the CIR can be obtained by

$$
\begin{aligned}
& E\left[h_{l}\left(n_{1}, \tau_{1}\right) h_{l}^{*}\left(n_{2}, \tau_{2}\right)\right] \\
& \quad=E\left[h_{l}\left(n_{1}, \tau_{1}\right) h_{l}^{*}\left(n_{2}, \tau_{2}\right)\right] \delta\left(\tau_{1}-\tau_{2}\right) \\
& \quad=\left.J_{0}\left(\beta \Delta_{n}\right) \sigma_{h_{\tau}}^{2}\right|_{\tau=\tau_{1}=\tau_{2}}, \quad 0 \leq \tau \leq \tau_{d},
\end{aligned}
$$

where $\delta(\cdot)$ is the Dirac delta function; $J_{0}(\cdot)$ is the zerothorder Bessel function of the first kind; $\Delta_{n} \triangleq n_{1}-n_{2} ; \sigma_{h_{\tau}}^{2}=$ $E\left[\left|h_{l}(\tau)\right|^{2}\right]$ is the power of the $\tau$ th channel tap; $\tau_{d}$ is the maximum delay spread of the channel; and $\beta=2 \pi f_{d} T / N$ where $f_{d}$ represents the maximum Doppler shift, $f_{d} T$ is the normalized Doppler frequency (NDF), $N$ is the number of subcarriers, and $T=N T_{S}$ is the symbol duration.

\section{ANALYSIS OF RECEIVED FREQUENCY-DOMAIN DATA AND SINR}

For convenience, let us define the start of the $l$ th symbol (excluding the CP with length $N_{G}$ ) at the time origin zero in the time coordinate. The estimated ST can be found to be located in one of the following three regions of an OFDM symbol: the Bad-ST1 region, the Good-ST region (also known as the ISI-free region), and the Bad-ST2 region in which the STOs are confined within the ranges of $-N_{G} \leq$ $n_{\delta} \leq-N_{G}+\tau_{d}-1,-N_{G}+\tau_{d} \leq n_{\delta} \leq 0$, and $1 \leq n_{\delta} \leq N-1$, respectively. Note that the first two regions are in the guard interval. Moreover, the transmitted signal of the $l$ th symbol can be represented as

$$
x_{l}(t)=\frac{1}{N} \sum_{m} X_{l, m} e^{j 2 \pi m t / N T_{S}}, \quad-N_{G} T_{S} \leq t<N T_{S},
$$

where $m$ is the transmitter subcarrier index. It is assumed that the symbol index $l$ is the same for both the receiver and the transmitter sides due to the ST and/or SCFO compensations. Consequently, after undergoing a multipath fading channel, the received signal can be determined as

$$
\tilde{x}_{l}(t)=\sum_{\tau=0}^{\tau_{d}} x_{l}\left(t-\tau T_{S}\right) h_{l}(t, \tau), \quad-N_{G} T_{S} \leq t<\left(N+\tau_{d}\right) T_{S},
$$

where $h_{l}(t, \tau)$ is the continuous time-selective CIR experienced by the $l$ th symbol. Then the overall received baseband signal, with the impairment of the CFO, can be written in the following summation form:

$$
\tilde{x}(t)=\sum_{l} \tilde{x}_{l}^{\prime}\left(t-l N_{S} T_{S}\right)+w^{\prime}(t)
$$

where $N_{S}=N+N_{G}$ is the OFDM symbol length including the CP,

$$
\begin{gathered}
\tilde{x}_{l}^{\prime}\left(t-l N_{S} T_{S}\right) \triangleq \tilde{x}_{l}\left(t-l N_{S} T_{S}\right) e^{j 2 \pi \varepsilon_{f} t / N T_{S}}, \\
w^{\prime}(t) \triangleq w(t) e^{j 2 \pi \varepsilon_{f} t / N T_{S}},
\end{gathered}
$$

and $w(t)$ is AWGN. In (4), the summation form can clearly describe the ISI effect between two consecutively received symbols when the ST is located in the Bad-ST regions.

The desired signal and interference (due to synchronization errors and time-selective channels) in the three different ST regions are separately analyzed as follows.

\subsection{Estimated ST located in good ST region}

In the Good-ST region, $n_{\delta}$ is within the range of $-N_{G}+\tau_{d} \leq$ $n_{\delta} \leq 0$. The received frequency-domain data, at the $k$ th subcarrier, are the FFT of the received time-domain data as written below

$$
\tilde{X}_{l, k, 0}=\operatorname{FFT}\left\{\left(\tilde{x}_{l, n^{\prime}}+w_{n^{\prime}}\right) g_{N}\left(n^{\prime}-n_{\delta}\right)\right\},
$$

where

$$
\tilde{x}_{l, n^{\prime}}=\left.\tilde{x}_{l}^{\prime}\left(t-l N_{S} T_{S}\right)\right|_{t=\left(l N_{S}+n^{\prime}\right) T_{S}^{\prime}}
$$

is the received $n^{\prime}$ th sample of the $l$ th symbol, FFT $\{\cdot\}$ is the FFT operation,

$$
g_{N}(n)= \begin{cases}1, & 0 \leq n<N \\ 0, & \text { otherwise }\end{cases}
$$

is the rectangular window function, and $w_{n^{\prime}} \triangleq$ $\left.w^{\prime}(t)\right|_{t=\left(l N_{S}+n^{\prime}\right) T_{S}}$ is the discrete-time AWGN. Note that the subscript 0 in (6) denotes the Good-ST region. With (2)-(5) and (7), the received frequency-domain data in (6), after some manipulations, can be found to be

$$
\tilde{X}_{l, k, 0}=\tilde{X}_{l, k, 0}^{d s r}+\tilde{N}_{k, 0},
$$

where

$$
\tilde{X}_{l, k, 0}^{d s r}=\hat{H}_{k, 0} X_{l, k} W_{N}^{\left[l N_{s}\left(k \varepsilon_{t}-\varepsilon_{f}\right)-k n_{\delta}\right]}
$$


is the desired signal, and

$$
\hat{N}_{k, 0} \triangleq \sum_{m \neq k} X_{l, m}\left[\frac{1}{N} \sum_{n^{\prime}=0}^{N-1} H_{l}\left(n^{\prime}, m\right) W_{N}^{n^{\prime} \phi_{m, k}}\right] W_{N}^{l N_{S}\left(m \varepsilon_{t}-\varepsilon_{f}\right)-k n_{\delta}}+v_{k}
$$

is the combined ICI and AWGN caused by the CFO, SCFO, AWGN, and doubly-selective channels. Note that in (10), the following notations are used: $W_{N} \triangleq e^{-j 2 \pi / N}$, and

$$
\hat{H}_{k, 0} \triangleq \frac{1}{N} \sum_{n^{\prime}=0}^{N-1} H_{l}\left(n^{\prime}, k\right) W_{N}^{-n^{\prime}\left(\varepsilon_{f}-k \varepsilon_{t}\right)}
$$

is the time-averaged time-selective frequency response of the channel where

$$
H_{l}\left(n^{\prime}, k\right) \triangleq \sum_{\tau=0}^{\tau_{d}} h_{l}\left(n^{\prime}, \tau\right) W_{N}^{k \tau}
$$

is the time-selective frequency response of the channel. Also note that in $(11), v_{k} \triangleq \operatorname{FFT}\left\{w_{n^{\prime}}\right\}$, and

$$
\phi_{m, k} \triangleq-m\left(1-\varepsilon_{t}\right)+k-\varepsilon_{f}
$$

is the normalized phase rotation which contains the CFO and SCFO effects. With (10) and (1), the desired signal power is derived in Appendix A and rewritten here for convenience:

$$
E\left[\left|\tilde{X}_{l, k, 0}^{d s r}\right|^{2}\right]=C \sigma_{X}^{2} \sum_{\Delta_{n}=1-N}^{N-1}\left(N-\left|\Delta_{n}\right|\right) J_{0}\left(\beta \Delta_{n}\right) W_{N}^{\Delta_{n} \phi_{k, k}}
$$

where $\sigma_{X}^{2}$ is the signal power and $C \triangleq \sum_{\tau=0}^{\tau_{d}} \sigma_{h_{\tau}}^{2} / N^{2}=\sigma_{H}^{2} / N^{2}$ is the total channel power normalized by $N^{2}$. Similarly, with (11) and (1), the power of combined ICI and AWGN can be shown to be

$$
\begin{aligned}
& E\left[\left|\hat{N}_{k, 0}\right|^{2}\right] \\
& \quad=C \sigma_{X}^{2} \sum_{m \neq k} \sum_{\Delta_{n}=1-N}^{N-1}\left(N-\left|\Delta_{n}\right|\right) J_{0}\left(\beta \Delta_{n}\right) W_{N}^{\Delta_{n} \phi_{m, k}}+\sigma_{v}^{2},
\end{aligned}
$$

where $\sigma_{v}^{2}$ is the AWGN power.

\subsection{Estimated ST located in bad-ST1 region}

In the Bad-ST1 region, $n_{\delta}$ is within the range of $-N_{G} \leq n_{\delta} \leq$ $-N_{G}+\tau_{d}-1$. Under this condition, the first $N_{1}=-N_{G}+$ $\tau_{d}-n_{\delta}$ samples received for the FFT operation are corrupted with the ISI incurred from the $(l-1)$ th symbol. Similar to (6), the received signal on the $k$ th subcarrier can be determined by separating the following $N$-point FFT into three different parts as

$$
\tilde{X}_{l, k, 1}=\tilde{X}_{l, k, 1}^{\prime}+\tilde{X}_{l, k, 1}^{\prime \prime}+\tilde{X}_{l, k, 1}^{\prime \prime \prime}+v_{k}
$$

Note that the subscript 1 denotes the Bad-ST1 region. The first part of (17)

$$
\begin{array}{r}
\tilde{X}_{l, k, 1}^{\prime}=\sum_{n^{\prime}=0}^{N_{1}-1}\left\{\left[\sum_{\tau=N_{G}+n^{\prime}+n_{\delta}+1}^{\tau_{d}}\left(\frac{1}{N} \sum_{m} X_{l-1, m} W_{N}^{-m\left[\psi_{l, n^{\prime}, n_{\delta}}-\left(l N_{S}+\tau\right)\right]}\right)\right.\right. \\
\left.\left.\times h_{l-1}\left(N_{S}+n_{\delta}+n^{\prime}, \tau\right)\right] W_{N}^{-\varepsilon_{f} \psi_{l, n^{\prime}, n_{\delta}}}\right\} W_{N}^{k n^{\prime}}
\end{array}
$$

is the $N$-point discrete Fourier transform (DFT) operated on the last $N_{1}$ output samples contributed by the linear convolution of the $(l-1)$ th symbol and the channel which results in ISI, where $\times$ denotes multiplication and

$$
\psi l, n^{\prime}, n_{\delta} \triangleq \frac{\left(l N_{S}+n^{\prime}+n_{\delta}\right) T_{S}^{\prime}}{T_{S}}
$$

The second part (which contributes to ICI)

$$
\begin{aligned}
\tilde{X}_{l, k, 1}^{\prime \prime}=\sum_{n^{\prime}=0}^{N_{1}-1}\left\{\left[\sum_{\tau=0}^{N_{G}+n^{\prime}+n_{\delta}}(\right.\right. & \left.\frac{1}{N} \sum_{m} X_{l, m} W_{N}^{-m\left[\psi_{l, n^{\prime}, n_{\delta}}-\left(l N_{S}+\tau\right)\right]}\right) \\
& \left.\left.\times h_{l}\left(n^{\prime}+n_{\delta}, \tau\right)\right] W_{N}^{-\varepsilon_{f} \psi_{l, n^{\prime}, n_{\delta}}}\right\} W_{N}^{k n^{\prime}}
\end{aligned}
$$

is the $N$-point DFT operated on the first $N_{1}$ samples, extracted by $g_{N}(n)$, from the linear convolution result of the $l$ th transmitted symbol's first $\tau_{d}$ samples with the CIR. The third part

$$
\begin{aligned}
\tilde{X}_{l, k, 1}^{\prime \prime \prime}=\sum_{n^{\prime}=N_{1}}^{N-1}\left\{\left[\sum_{\tau=0}^{\tau_{d}}\right.\right. & \left(\frac{1}{N} \sum_{m} X_{l, m} W_{N}^{-m\left[\psi_{l, n^{\prime}, n_{\delta}}-\left(l N_{S}+\tau\right)\right]}\right) \\
& \left.\left.\times h_{l}\left(n^{\prime}+n_{\delta}, \tau\right)\right] W_{N}^{-\varepsilon_{f} \psi_{l, n^{\prime}, n_{\delta}}}\right\} W_{N}^{k n^{\prime}}
\end{aligned}
$$

is the $N$-point DFT operated on the remaining $N-N_{1}$ samples from the circular convolution result of the $l$ th transmitted symbol's $N-N_{1}$ samples (i.e., from the $\left(-N_{G}+\right.$ $\left.\tau_{d}\right)$ th to the $\left(N-n_{\delta}-1\right)$ th samples) with the CIR. The remaining derivation is detailed in Appendix B, and final results are rewritten here:

$$
\begin{aligned}
& E\left[\left|\tilde{X}_{l, k, 1}^{d s r}\right|^{2}\right] \\
& \quad=C \sigma_{X}^{2} \sum_{\Delta_{n}=-\left(N-N_{1}-1\right)}^{N-N_{1}-1}\left(N-N_{1}-\left|\Delta_{n}\right|\right) J_{0}\left(\beta \Delta_{n}\right) W_{N}^{\phi_{k, k} \Delta_{n}}
\end{aligned}
$$


is the desired signal power, and

$$
\begin{aligned}
& E\left[\left|\tilde{N}_{k, 1}\right|^{2}\right] \\
& =C \sigma_{X}^{2} \sum_{m \neq k} \sum_{\Delta_{n}=-\left(N-N_{1}-1\right)}^{N-N_{1}-1}\left(N-N_{1}-\left|\Delta_{n}\right|\right) J_{0}\left(\beta \Delta_{n}\right) W_{N}^{\phi_{m, k} \Delta_{n}} \\
& \quad+C \sigma_{X}^{2} \sum_{m} \sum_{\Delta_{n}=-\left(N_{1}-1\right)}^{N_{1}-1}\left(N_{1}-\left|\Delta_{n}\right|\right) J_{0}\left(\beta \Delta_{n}\right) W_{N}^{\phi_{m, k} \Delta_{n}} \\
& \quad+2 \frac{\sigma_{X}^{2}}{N^{2}} \sum_{m \neq k} \sum_{n_{1}=N_{1}}^{N-1} \sum_{n_{2}=0}^{N_{1}-1} J_{0}\left(\beta \Delta_{n}\right) W_{N}^{\phi_{m, k} \Delta_{n}} \sum_{\tau=0}^{n_{\delta}+N_{G}+n_{2}} \sigma_{h_{\tau}}^{2}+\sigma_{v}^{2}
\end{aligned}
$$

is the power of the combined interference (including ISI and ICI) and AWGN.

\subsection{Estimated ST located in bad-ST2 region}

In the Bad-ST2 region, $n_{\delta}$ is within the range of $1 \leq n_{\delta} \leq N-$ 1. Since the derivation is similar to Section 3.2, it is omitted here. The desired signal power can be found to be

$$
\begin{aligned}
& E\left[\left|\tilde{X}_{l, k, 2}^{d s r}\right|^{2}\right] \\
& =C \sigma_{X}^{2} \sum_{\Delta_{n}=-\left(N-n_{\delta}-1\right)}^{N-n_{\delta}-1}\left(N-n_{\delta}-\left|\Delta_{n}\right|\right) J_{0}\left(\beta \Delta_{n}\right) W_{N}^{\phi_{k, k} \Delta_{n}}, \\
& E\left[\left|\tilde{N}_{k, 2}\right|^{2}\right] \\
& =C \sigma_{X}^{2} \sum_{m \neq k} \sum_{\Delta_{n}=-\left(N-n_{\delta}-1\right)}^{N-n_{\delta}-1}\left(N-n_{\delta}-\left|\Delta_{n}\right|\right) J_{0}\left(\beta \Delta_{n}\right) W_{N}^{\phi_{m, k} \Delta_{n}} \\
& +C \sigma_{X}^{2} \sum_{m} \sum_{\Delta_{n}=-\left(n_{\delta}-1\right)}^{n_{\delta}-1}\left(n_{\delta}-\left|\Delta_{n}\right|\right) J_{0}\left(\beta \Delta_{n}\right) W_{N}^{\phi_{m, k} \Delta_{n}}+2 \frac{\sigma_{X}^{2}}{N^{2}} \\
& \quad \times \sum_{m \neq k}^{N-n_{\delta}-1} \sum_{n_{1}=0}^{N-1} \sum_{n_{2}=N-n_{\delta}}^{N-1} J_{0}\left(\beta \Delta_{n}\right) W_{N}^{\phi_{m, k} \Delta_{n}} \sum_{\tau=-N+n_{\delta}+n_{2}+1}^{\tau_{d}} \sigma_{h_{\tau}}^{2}+\sigma_{v}^{2}
\end{aligned}
$$

is the power of the combined interference and AWGN.

\subsection{SINR analysis}

Finally, based on the results in Sections 3.1 to 3.3, the SINR can be written as

$$
\eta_{k, r}=\frac{E\left[\left|\tilde{X}_{l, k, r}^{d s r}\right|^{2}\right]}{E\left[\left|\tilde{N}_{k, r}\right|^{2}\right]}
$$

where $r=0,1,2$ denotes those three different ST regions.

As shown in Appendix C, an interesting observation is that the ICI powers (16) are approximately the same when $f_{d} T=\sqrt{2} \varepsilon_{f}$. It can be easily verified that this is also true for the desired signal power in all of the three ST regions. So are the SINRs.

\section{MORE COMPACT RESULTS}

By utilizing the fact that

$$
\sum_{m \neq k} W_{N}^{-\Delta_{n}(m-k)}= \begin{cases}-1, & \Delta_{n} \neq 0 \\ N-1, & \Delta_{n}=0,\end{cases}
$$

and both (24) and (25) are even functions of $\Delta_{n}$, given that the SCFO is negligible, one can reduce (24) and (25) to a more simpler form as

$$
\begin{aligned}
E\left[\left|\tilde{X}_{l, k, r}^{d s r}\right|^{2}\right] & C \sigma_{X}^{2}\left(N-n_{\delta}\right)+2 C \sigma_{X}^{2} \sum_{\Delta_{n}=1}^{N-n_{\delta}-1}\left(N-n_{\delta}-\Delta_{n}\right) \\
& \times J_{0}\left(\beta \Delta_{n}\right) \cos \left(\frac{2 \pi \Delta_{n} \varepsilon_{f}}{N}\right), \\
E & {\left[\left|\tilde{N}_{k, r}\right|^{2}\right] } \\
= & C \sigma_{X}^{2}\left[N(N-1)+n_{\delta}\right]-2 C \sigma_{X}^{2} \\
& \times \sum_{\Delta_{n}=1}^{N-n_{\delta}-1}\left(N-n_{\delta}-\Delta_{n}\right) J_{0}\left(\beta \Delta_{n}\right) \cos \left(\frac{2 \pi \Delta_{n} \varepsilon_{f}}{N}\right) \\
& -2 \frac{\sigma_{X}^{2}}{N^{2}} \sum_{n_{1}=0}^{N-n_{\delta}-1} \sum_{n_{2}=N-n_{\delta}}^{N-1} J_{0}\left(\beta \Delta_{n}\right) W_{N}^{-\Delta_{n} \varepsilon_{f}} \sum_{\tau=-N+n_{\delta}+n_{2}+1}^{\tau_{d}} \sigma_{h_{\tau}}^{2}+\sigma_{v}^{2} .
\end{aligned}
$$

It is shown that both compact forms are independent of the subcarrier index. By contrast, the SINR depends on the subcarrier index under the influence of the SCFO. Note that this result can be applied to the cases of $r=0$ (by setting $\left.n_{\delta}=0\right)$ and $r=2$.

To gain further insight into (28), the SIR $\rho$, under the influence of STO alone, and the influence of combined CFO and NDF, can be respectively reduced to

$$
\begin{aligned}
\rho_{\text {STO }} & =\frac{\left(N-n_{\delta}\right)^{2}}{\left(2 N-n_{\delta}\right) n_{\delta}-2\left(\left(N-n_{\delta}\right) / \sigma_{H}^{2}\right) X}, \\
f_{d} T & =\varepsilon_{f}=0,
\end{aligned}
$$

where $\mathcal{X}$ denotes $\sum_{n_{2}=N-n_{\delta}}^{N-1} \sum_{\tau=-N+n_{\delta}+n_{2}+1}^{\tau_{d}} \sigma_{h_{\tau}}^{2}$, and

$$
\rho_{\mathrm{CFO} \& \mathrm{NDF}}=\frac{N+2 \mathcal{y}}{N(N-1)-2 \mathcal{y}}, \quad n_{\delta}=0,
$$

where $y$ denotes $\sum_{\Delta_{n}=1}^{N-1}\left(N-\Delta_{n}\right) J_{0}\left(\beta \Delta_{n}\right) \cos \left(2 \pi \Delta_{n} \varepsilon_{f} / N\right)$.

Note that based on our derivation, the result in $[1$, Equation (17)] can be further reduced to a more concise form as (30), and the result in [2, Equation (2)] is the same as (29).

With (30) and Taylor's series of the cosine function, after some manipulations and the fact that $N^{2} \gg 1$, the SINR under the influence of the CFO can be shown to be

$$
\eta_{\mathrm{CFO}} \approx \frac{6-2 \pi^{2}\left(\varepsilon_{f}\right)^{2}}{\pi^{2}\left(\varepsilon_{f}\right)^{2}+6 / \gamma}, \quad f_{d} T=n_{\delta}=0,
$$

where $\gamma$ is SNR. 


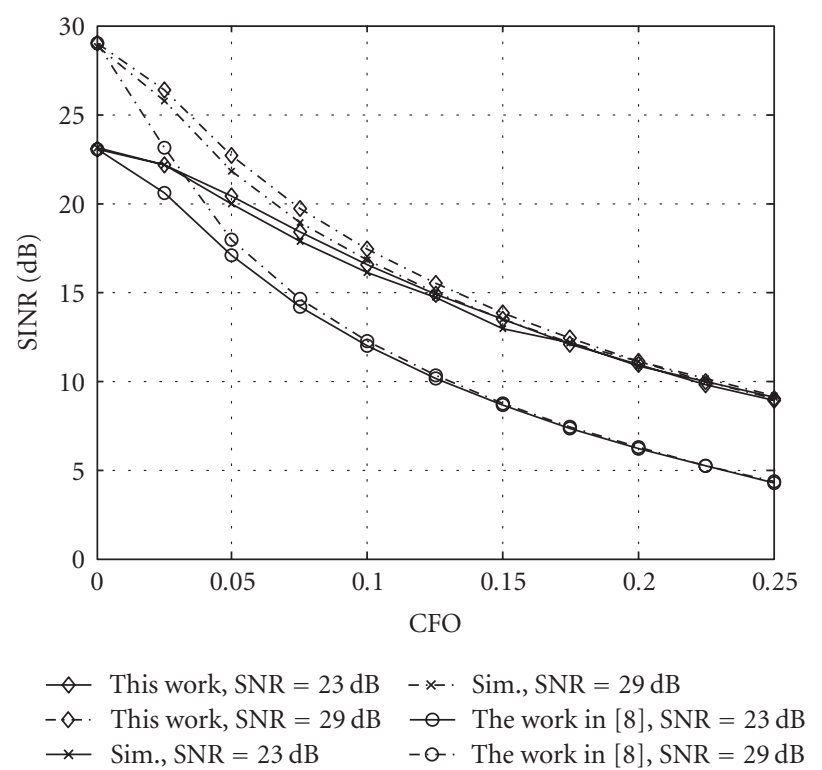

Figure 2: SINR plotted against CFO, under SNR $=23$ and $29 \mathrm{dBs}$.

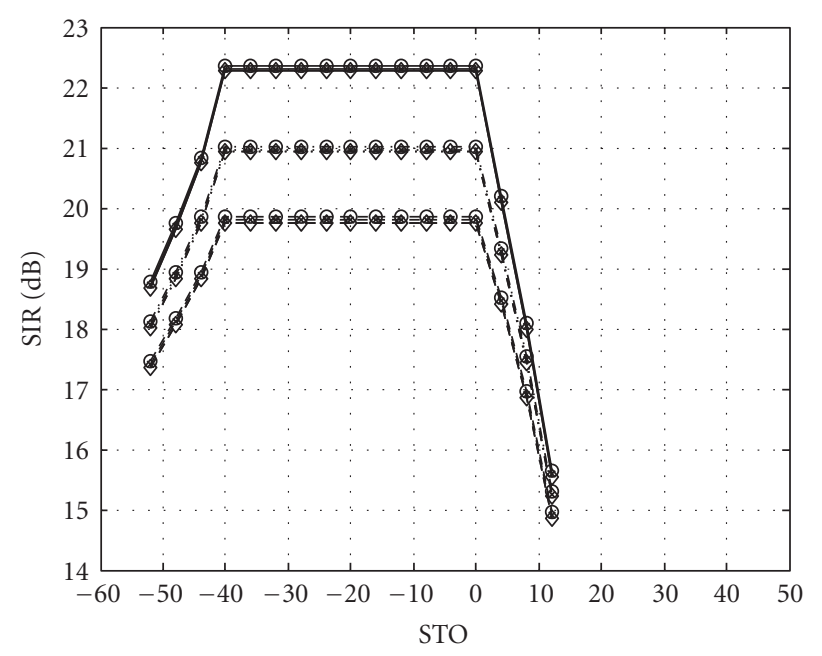

$$
\begin{aligned}
& \text { - Anal. } f_{d} T=0.06 \quad-\quad \text { Sim. } f_{d} T=0.06 \\
& \text {-. Anal. } f_{d} T=0.07 \quad-0 \text {. Sim. } f_{d} T=0.07 \\
& -- \text { Anal. } f_{d} T=0.08 \quad-\Theta-\text { Sim. } f_{d} T=0.08 \\
& \multimap \text { Anal. } \varepsilon_{f}=0.0424 \quad \rightarrow \quad \text { Sim. } \varepsilon_{f}=0.0424 \\
& -\diamond \text {. Anal. } \varepsilon_{f}=0.0495 \quad-x-\text {. Sim. } \varepsilon_{f}=0.0495 \\
& -\diamond-\text { Anal. } \varepsilon_{f}=0.0566 \quad-\star-\text { Sim. } \varepsilon_{f}=0.0566
\end{aligned}
$$

FIGURE 3: SIR plotted against STO, under the influences of the CFO and NDF.

To verify the concise result of (31), the SINR as a function of the CFO is shown in Figure 2. The result in $[10$, Equation (15)] and the simulation result are also included for validation, assuming quadrature phase-shift keying (QPSK) modulation, $N=256, \gamma=23$ and $29 \mathrm{dBs}$. As can be seen, the derived result (31) is more accurate than that in [10, Equation (15)].

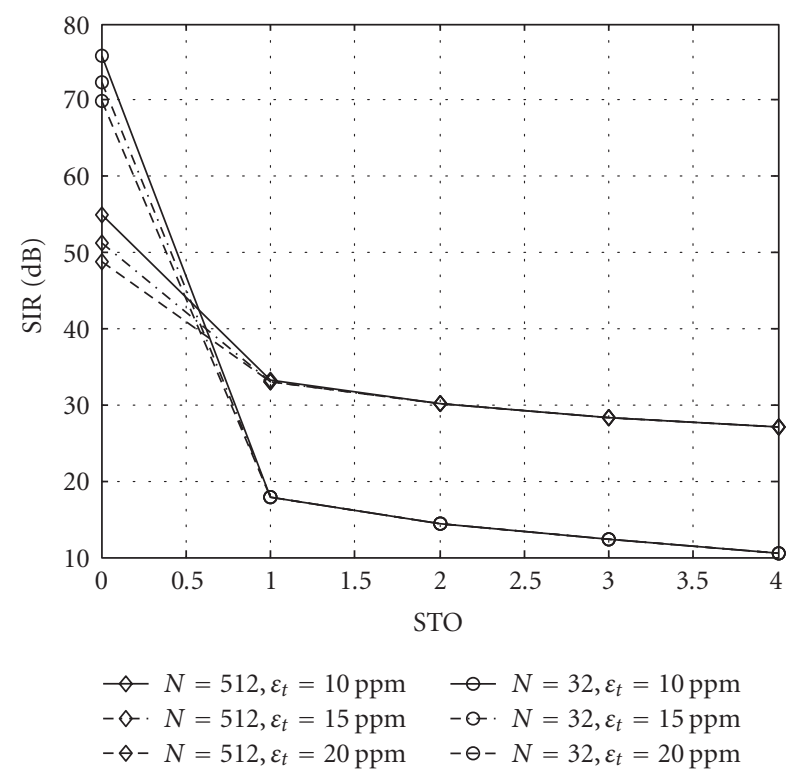

FIGURE 4: SIR plotted against STO under the influence of the SCFO. $\mathrm{NDF}=\mathrm{CFO}=0$. Subcarrier index $=6$.

\section{NUMERICAL AND SIMULATION RESULTS}

In the simulation, an OFDM system, with $N=256$ subcarriers and a guard interval of $N_{G}=N / 8=32$ samples, is considered. The adopted modulation scheme is QPSK. The signal bandwidth is $2.5 \mathrm{MHz}$, and the radio frequency is $2.4 \mathrm{GHz}$. The subcarrier spacing is $8.68 \mathrm{kHz}$. The OFDM symbol duration is $115.2 \mu$ s. The maximum delay spread $\tau_{d}$ of the channel is 24 samples. The channel taps are randomly generated by independent zero-mean unitvariance complex Gaussian variables with $\sum_{\tau} E\left\{\left|h_{l}(\tau)\right|^{2}\right\}=$ 1 for each simulation run. In each simulation run, 10000 OFDM symbols are tested. The same channels are used for both the numerical and simulation analyses. All the results are obtained by averaging over 2000 independent channel realizations.

The following example demonstrates some design constraints to achieve the typical condition of SIR $>20 \mathrm{~dB}$. The SIR curves under the joint effects of the STO, NDF, and $\mathrm{CFO}$ are shown in Figure 3. As shown, for the condition of SIR $>20 \mathrm{~dB}$ to be satisfied, the NDF should be less than $8 \%$ as observed in [1], and the CFO should be less than $6 \%$. This figure also shows that the SIRs are the same when $f_{d} T=$ $\sqrt{2} \varepsilon_{f}$. To achieve SIR $>20 \mathrm{~dB}$, the STO, when $f_{d} T=0.00$, should be less than 8 samples.

As can be seen in Figure 3, the SIRs are $22.2 \mathrm{~dB}$ and $20.9 \mathrm{~dB}$ due to the single error of $\mathrm{NDF}=0.06$ and $\mathrm{STO}=6$ (samples), respectively. However, when both errors of $\mathrm{NDF}=0.06$ and STO $=6$ coexist, the SIR drops to $18.5 \mathrm{~dB}$. The degradation due to the combined synchronization errors is $3.7 \mathrm{~dB}$ more than the single error of NDF, while $2.4 \mathrm{~dB}$ more than the single error of STO. Therefore, the degradation of the SINR due to the combined synchronization errors may be much more severe than a single synchronization error. 
The SIR curves under the joint effects of the STO and SCFO are shown in Figure 4. When the $\mathrm{STO}=0$, and under the same SCFO condition, the SIR deteriorates as $N$ increases; on the contrary, when the $\mathrm{STO} \neq 0$, the SIR also decreases as $N$ decreases, because there are less numbers of subcarriers. In other words, the impact on performance due to the STO is more apparent for a smaller $N$ than a larger $N$. It can also be seen that the SCFO has a very minor effect on the SIR. Moreover, effect of the STO is much more significant than that of the SCFO.

\section{CONCLUSION}

The impacts of the combined synchronization errors have been analyzed. It has been found that the NDF and CFO have the same impacts on the SIR when $f_{d} T=\sqrt{2} \varepsilon_{f}$. Due to impairments of the synchronization algorithms, the tolerance regarding those synchronization errors should be taken into consideration, especially in a mobile environment. In addition, it has also been found that the effect of the combined synchronization errors on the SINR may be much more severe than a single synchronization error. Therefore, it is beneficial to study the effects of combined synchronization errors. The derived results can be used as design guidelines for devising suitable synchronization algorithms in doublyselective fading channels.

\section{APPENDICES}

\section{A. DERIVATION OF THE SIGNAL POWER OF (15) FOR THE GOOD-ST REGION IN SECTION 3.1}

Since the channel fading characteristic is independent of the transmitted data, the signal power (15) can be found to be

$$
\begin{aligned}
& E\left[\left|\tilde{X}_{l, k, 0}^{d s r}\right|^{2}\right] \\
& =\frac{1}{N^{2}} E\left[\left|X_{l, k}\right|^{2}\right] \sum_{n_{1}=0}^{N-1} \sum_{n_{2}=0}^{N-1} E\left[H_{l}\left(n_{1}, k\right) H_{l}\left(n_{2}, k\right)^{*}\right] W_{N}^{\phi_{k, k}\left(n_{1}-n_{2}\right)} .
\end{aligned}
$$

With (13) and (1), the correlation of the time-selective transfer function of the channel in (A.1) can be found to be

$$
E\left[H_{l}\left(n_{1}, k\right) H_{l}\left(n_{2}, k\right)^{*}\right]=J_{0}\left(\beta\left(n_{1}-n_{2}\right)\right) \sum_{\tau=0}^{\tau_{d}} \sigma_{h_{\tau}}^{2}
$$

Finally, by inserting (A.2) into (A.1), and knowing that $\Delta_{n}=$ $n_{1}-n_{2}$, the signal power can be shown to be

$$
E\left[\left|\tilde{X}_{l, k, 0}^{d s r}\right|^{2}\right]=C \sigma_{X}^{2} \sum_{\Delta_{n}=1-N}^{N-1}\left(N-\left|\Delta_{n}\right|\right) J_{0}\left(\beta \Delta_{n}\right) W_{N}^{\phi_{k, k} \Delta_{n}},
$$

where $\sigma_{X}^{2}$ is the transmitted signal power and $C \triangleq \sum_{\tau=0}^{\tau_{d}} \sigma_{h_{\tau}}^{2} /$ $N^{2}=\sigma_{H}^{2} / N^{2}$ is the total channel power normalized by $N^{2}$.

\section{B. DETAILED DERIVATION OF SIGNAL AND INTERFERENCE POWERS FOR THE BAD-ST1 REGION IN SECTION 3.2}

From (17), we can separate the desired signal, and the combined interference and AWGN as

$$
\tilde{X}_{l, k, 1}=\tilde{X}_{l, k, 1}^{d s r}+\tilde{N}_{k, 1}
$$

where

$$
\tilde{X}_{l, k, 1}^{d s r}=\hat{H}_{k, 1} X_{l, k} W_{N}^{\left[l N_{s}\left(k \varepsilon_{t}-\varepsilon_{f}\right)-k n_{\delta}\right]}
$$

is the desired data,

$$
\hat{H}_{k, 1} \triangleq \frac{1}{N} \sum_{n^{\prime}=N_{1}}^{N-1} H_{l}\left(n^{\prime}+n_{\delta}, k\right) W_{N}^{-n^{\prime}\left(\varepsilon_{f}-k \varepsilon_{t}\right)}
$$

is the time-averaged time-selective transfer function of the channel, and

$$
\tilde{N}_{k, 1}=\tilde{X}_{l, k, 1}^{\prime}+\tilde{X}_{l, k, 1}^{\prime \prime}+\left(\tilde{X}_{l, k, 1}^{\prime \prime \prime}-\tilde{X}_{l, k, 1}^{d s r}\right)+v_{k}
$$

is the combined interference (caused by the STO, CFO, SCFO, and time-selective channels) and AWGN. With (B.2), (B.3), (13), and (1), it can be shown that

$$
\begin{aligned}
& E\left[\left|\tilde{X}_{l, k, 1}^{d s r}\right|^{2}\right] \\
& \quad=C \sigma_{X}^{2} \sum_{\Delta_{n}=-\left(N-N_{1}-1\right)}^{N-N_{1}-1}\left(N-N_{1}-\left|\Delta_{n}\right|\right) J_{0}\left(\beta \Delta_{n}\right) W_{N}^{\phi_{k, k} \Delta_{n}} .
\end{aligned}
$$

Since transmitted data of different symbols are independent, the power of the combined interference and AWGN can be determined as

$$
\begin{aligned}
& E\left[\left|\tilde{N}_{k, 1}\right|^{2}\right] \\
& =E\left[\left|\tilde{X}_{l, k, 1}^{\prime \prime}\right|^{2}\right]+E\left[\left|\tilde{X}_{l, k, 1}^{\prime \prime \prime}-\tilde{X}_{l, k, 1}^{d s r}\right|^{2}\right] \\
& \quad+2 E\left[\tilde{X}_{l, k, 1}^{\prime \prime}\left(\tilde{X}_{l, k, 1}^{\prime \prime \prime}-\tilde{X}_{l, k, 1}^{d s r}\right)^{*}\right]+E\left[\left|\tilde{X}_{l, k, 1}^{\prime}\right|^{2}\right]+\sigma_{v}^{2} .
\end{aligned}
$$

After some manipulations, it can be shown that

$$
\begin{aligned}
& E\left[\left|\tilde{X}_{l, k, 1}^{\prime \prime}\right|^{2}\right]+E\left[\left|\tilde{X}_{l, k, 1}^{\prime}\right|^{2}\right] \\
& =C \sigma_{X}^{2} \sum_{m} \sum_{\Delta_{n}=-\left(N_{1}-1\right)}^{N_{1}-1}\left(N_{1}-\left|\Delta_{n}\right|\right) J_{0}\left(\beta \Delta_{n}\right) W_{N}^{\phi_{m, k} \Delta_{n}}, \\
& E\left[\left|\tilde{X}_{l, k, 1}^{\prime \prime \prime}-\tilde{X}_{l, k, 1}^{d s r}\right|^{2}\right] \\
& =C \sigma_{X}^{2} \sum_{m \neq k} \sum_{\Delta_{n}=-\left(N-N_{1}-1\right)}^{N-N_{1}-1}\left(N-N_{1}-\left|\Delta_{n}\right|\right) J_{0}\left(\beta \Delta_{n}\right) W_{N}^{\phi_{m, k} \Delta_{n}}, \\
& E\left[\tilde{X}_{l, k, 1}^{\prime \prime}\left(\tilde{X}_{l, k, 1}^{\prime \prime \prime}-\tilde{X}_{l, k, 1}^{d s r}\right)^{*}\right] \\
& \quad=\frac{\sigma_{X}^{2}}{N^{2}} \sum_{m \neq k} \sum_{n_{1}=N_{1}}^{N-1} \sum_{n_{2}=0}^{N_{1}-1} J_{0}\left(\beta \Delta_{n}\right) W_{N}^{\phi_{m, k} \Delta_{n}} \sum_{\tau=0}^{n_{\delta}+N_{G}+n_{2}} \sigma_{h_{\tau}}^{2} .
\end{aligned}
$$


Finally, by inserting (B.7) into (B.6), the power of the combined interference and AWGN can be written as

$$
\begin{aligned}
& E\left[\left|\tilde{N}_{k, 1}\right|^{2}\right] \\
& =C \sigma_{X}^{2} \sum_{m \neq k} \sum_{\Delta_{n}=-\left(N-N_{1}-1\right)}^{N-N_{1}-1}\left(N-N_{1}-\left|\Delta_{n}\right|\right) J_{0}\left(\beta \Delta_{n}\right) W_{N}^{\phi_{m, k} \Delta_{n}} \\
& +C \sigma_{X}^{2} \sum_{m} \sum_{\Delta_{n}=-\left(N_{1}-1\right)}^{N_{1}-1}\left(N_{1}-\left|\Delta_{n}\right|\right) J_{0}\left(\beta \Delta_{n}\right) W_{N}^{\phi_{m, k} \Delta_{n}} \\
& +2 \frac{\sigma_{X}^{2}}{N^{2}} \sum_{m \neq k} \sum_{n_{1}=N_{1}}^{N-1} \sum_{n_{2}=0}^{N_{1}-1} J_{0}\left(\beta \Delta_{n}\right) W_{N}^{\phi_{m, k}} \sum_{\tau=0}^{n_{\delta}+N_{G}+n_{2}} \sigma_{h_{\tau}}^{2}+\sigma_{v}^{2} .
\end{aligned}
$$

\section{THE RELATIONSHIP OF THE NDF AND CFO THAT EXHIBITS THE SAME ICI POWER IN (16)}

In the following, we will find the condition when NDF has the same impact on the ICI power with the CFO.

With (16), the ICI powers under the influence of the NDF (without the $\mathrm{CFO}$ ) and $\mathrm{CFO}$ (without the NDF) are

$$
\begin{gathered}
C \sigma_{X}^{2} \sum_{\Delta_{n}=-(N-1)}^{N-1}\left(N-\left|\Delta_{n}\right|\right) J_{0}\left(\beta \Delta_{n}\right) W_{N}^{-\Delta_{n}\left[m\left(1-\varepsilon_{t}\right)-k\right]}, \\
C \sigma_{X}^{2} \sum_{\Delta_{n}=-(N-1)}^{N-1}\left(N-\left|\Delta_{n}\right|\right) W_{N}^{-\Delta_{n} \varepsilon_{f}} W_{N}^{-\Delta_{n}\left[m\left(1-\varepsilon_{t}\right)-k\right]},
\end{gathered}
$$

respectively. When (C.1) equals (C.2), the zeroth-order Bessel function of the first kind $J_{0}(\cdot)$ has the same value with the complex exponential function $W_{N}=e^{-j 2 \pi / N}$. In addition, the Taylor series of the zeroth-order Bessel function of the first kind and the complex exponential function are

$$
\begin{gathered}
J_{0}\left(x_{1}\right)=1-\frac{\left(x_{1} / 2\right)^{2}}{(1 !)^{2}}+\frac{\left(x_{1} / 2\right)^{4}}{(2 !)^{2}}-\frac{\left(x_{1} / 2\right)^{6}}{(3 !)^{2}}+\cdots, \\
e^{\left(x_{2}\right)}=1+\frac{x_{2}}{1 !}+\frac{x_{2}^{2}}{2 !}+\frac{x_{2}^{3}}{3 !}+\cdots,
\end{gathered}
$$

respectively, where $x_{1}=2 \pi f_{d} T \Delta_{n} / N$ and $x_{2}=j 2 \pi \Delta_{n} \varepsilon_{f} / N$. Since $\Delta_{n}$ ranges from $-(N-1)$ to $N-1$, the odd power terms (and pure imaginary) of (C.4) will be cancelled when they are inserted into (C.2). Furthermore, since $f_{d} T$ and $\varepsilon_{f}$ are typically less than $10^{-1},(\mathrm{C} .3$ ) and (C.4) can be well approximated by the first two terms. As a result, the condition of $(\mathrm{C} .1)=(\mathrm{C} .2)$ implies that

$$
1-\frac{\left(x_{1} / 2\right)^{2}}{(1 !)^{2}}=1+\frac{x_{2}^{2}}{2 !}
$$

which leads to the result of $f_{d} T=\sqrt{2} \varepsilon_{f}$.

\section{SUMMARY OF NOTATIONS}

Since there are so many notations used in this work, for clarity, the notations are collectively defined and summarized in this section. Please note that subscripts $l, r, k$ (or $m$ ), $n$ denote the $l$ th symbol, $r$ th ST region, $k$ th (or $m$ th) subcarrier, and $n$th sample, respectively. $\delta(\cdot): \quad$ Dirac delta function

$\eta_{k, r}: \quad$ SINR

$\rho: \quad$ Signal-to-interference ratio (SIR)

$\gamma: \quad$ Signal-to-noise ratio (SNR)

$\sigma_{h_{\tau}}^{2}: \quad$ Power of the $\tau$ th channel tap

$\sigma_{v}^{2}: \quad$ Additive white Gaussian noise (AWGN) power

$\sigma_{X}^{2}$ : Transmitted signal power

$\Delta_{n}: \quad$ Time difference

$\tau_{d}: \quad$ Maximum delay spread of the channel

$\tau: \quad$ Path delay of the channel

$\beta: \quad \triangleq 2 \pi f_{d} T / N$

$\phi_{m, k}$ : Normalized phase rotation which contains the $\mathrm{CFO}$ and SCFO effects

$(\cdot)_{N}: \quad$ Modulo $N$ operation

$\varepsilon_{f}: \quad \mathrm{CFO}$

$\varepsilon_{t}: \quad$ SCFO

$\cos (\cdot)$ Cosine function

C: $\quad \triangleq \sum_{\tau=0}^{\tau_{d}} \sigma_{h_{\tau}}^{2} / N^{2}=\sigma_{H}^{2} / N^{2}$, total channel power normalized by $N^{2}$

$f_{c}: \quad$ Carrier frequency

$f_{d}$ : Maximum Doppler shift in Hertz

FFT $\{\cdot\}$ : Fast Fourier transform (FFT) operation

$g_{N}(n): \quad$ Rectangular window function

$h_{l}(n, \tau): \quad \tau$ th channel tap of the discrete time-variant channel impulse responses (CIR)

$h_{l}(t, \tau): \quad \tau$ th channel tap of the continuous-time timevariant channel impulse responses (CIR)

$\hat{H}_{k, r}$ : Time-averaged time-variant transfer function of the channel

$H_{l}(n, m)$ : Time-variant transfer function of the channel

$J_{0}(\cdot)$ : Zeroth-order Bessel function of the first kind

$n_{\delta}: \quad$ STO

$N$ : $\quad$ Number of subcarriers

$N_{G}: \quad$ Cyclic prefix (CP) length

$N_{S}$ : $\quad$ OFDM symbol length including the CP

$\hat{N}_{k, r}$ : Combined interference and AWGN

$N_{1}$ : $\quad$ Length of corrupted samples when the symbol time is located in the Bad-ST1 region (please see Section 3.2)

T: $\quad$ Symbol duration including the CP

$1 / T_{S}$ : Transmitter's sampling frequency

$1 / T_{S}^{\prime}: \quad$ Receiver's sampling frequency

$v_{k}: \quad \quad \quad$ AWGN at the $k$ th subcarrier

$w(t)$ : Continuous-time AWGN

$w^{\prime}(t): \quad$ AWGN affected by the CFO

$w_{n^{\prime}}: \quad$ Discrete-time AWGN

$W_{N}: \quad \triangleq e^{-j 2 \pi / N}$, twiddle factor

$x_{l}(t): \quad$ Transmitted time-domain signal

$\tilde{x}_{l}(t)$ : Time-domain signal under the influence of the channel

$\tilde{x}_{l}^{\prime}(t): \quad$ Time-domain signal under the influence of CFO

$\tilde{x}(t): \quad$ Overall received baseband signal

$\tilde{x}_{l, n^{\prime}}: \quad$ Received time-domain data

$X_{l, k}: \quad$ Transmitted frequency-domain data

$\tilde{X}_{l, k, r}: \quad$ Received frequency-domain data

$\tilde{X}_{l, k, r}^{d s r}: \quad$ Desired signal. 


\section{ACKNOWLEDGMENT}

The authors would like to thank the editor and anonymous reviewers for their helpful comments and suggestions in improving the quality of this paper.

\section{REFERENCES}

[1] J. Li and M. Kavehrad, "Effects of time selective multipath fading on OFDM systems for broadband mobile applications," IEEE Communications Letters, vol. 3, no. 12, pp. 332-334, 1999.

[2] Y. Mostofi and D. C. Cox, "Mathematical analysis of the impact of timing synchronization errors on the performance of an OFDM system," IEEE Transactions on Communications, vol. 54, no. 2, pp. 226-230, 2006.

[3] M. Park, K. Ko, H. Yoo, and D. Hong, "Performance analysis of OFDMA uplink systems with symbol timing misalignment," IEEE Communications Letters, vol. 7, no. 8, pp. 376-378, 2003.

[4] I. R. Capoglu, Y. Li, and A. Swami, "Effect of Doppler spread in OFDM-based UWB systems," IEEE Transactions on Wireless Communications, vol. 4, no. 5, pp. 2559-2567, 2005.

[5] B. Stantchev and G. Fettweis, "Time-variant distortions in OFDM," IEEE Communications Letters, vol. 4, no. 10, pp. 312$314,2000$.

[6] H. Steendam and M. Moeneclaey, "Analysis and optimization of the performance of OFDM on frequency-selective timeselective fading channels," IEEE Transactions on Communications, vol. 47, no. 12, pp. 1811-1819, 1999.

[7] H. Steendam and M. Moeneclaey, "Synchronization sensitivity of multicarrier systems," European Transactions on Telecommunications, vol. 15, no. 3, pp. 223-234, 2004.

[8] M. Speth, S. Fechtel, G. Fock, and H. Meyr, "Optimum receiver design for OFDM-based broadband transmissionpart II: a case study," IEEE Transactions on Communications, vol. 49, no. 4, pp. 571-578, 2001.

[9] M. S. El-Tanany, Y. Wu, and L. Házy, "OFDM uplink for interactive broadband wireless: analysis and simulation in the presence of carrier, clock and timing errors," IEEE Transactions on Broadcasting, vol. 47, no. 1, pp. 3-19, 2001.

[10] P. H. Moose, "Technique for orthogonal frequency division multiplexing frequency offset correction," IEEE Transactions on Communications, vol. 42, no. 10, pp. 2908-2914, 1994.

[11] Z. Cao, U. Tureli, and Y.-D. Yao, "Low-complexity orthogonal spectral signal construction for generalized OFDMA uplink with frequency synchronization errors," IEEE Transactions on Vehicular Technology, vol. 56, no. 3, pp. 1143-1154, 2007.

[12] J. Choi, C. Lee, H. W. Jung, and Y. H. Lee, "Carrier frequency offset compensation for uplink of OFDM-FDMA systems," IEEE Communications Letters, vol. 4, no. 12, pp. 414-416, 2000.

[13] M.-O. Pun, M. Morelli, and C.-C. J. Kuo, "Maximumlikelihood synchronization and channel estimation for OFDMA uplink transmissions," IEEE Transactions on Communications, vol. 54, no. 4, pp. 726-736, 2006.

[14] M. Morelli, "Timing and frequency synchronization for the uplink of an OFDMA system," IEEE Transactions on Communications, vol. 52, no. 2, pp. 296-306, 2004.

[15] A. M. Tonello, N. Laurenti, and S. Pupolin, "Analysis of the uplink of an asynchronous multi-user DMT OFDMA system impaired by time offsets, frequency offsets, and multi-path fading," in Proceedings of the 52nd IEEE Vehicular Technology Conference (VTC '00), vol. 3, pp. 1094-1099, Boston, Mass, USA, September 2000.

[16] W. C. Jakes, Ed., Microwave Mobile Communications, John Wiley \& Sons, New York, NY, USA, 1974. 